\title{
2,3,4,5-Tetrahydro-2,4-diimino-1,3,5-triazin-1-ium salts in the reaction of carbodiimides with $\mathrm{N}$-alkylnitrilium salts
}

\author{
Rajab Abu-El-Halawa \\ Al al-Bayt University, Chemistry Department, Mafraq, Jordan \\ E-mail: halawarajab@yahoo.com
}

\begin{abstract}
2,3,4,5-Tetrahydro-2,4-diimino-1,3,5-triazin-1-ium hexachloroantimonate salts were formed by the addition of carbodiimides to $\mathrm{N}$-alkylnitrilium salts. The compounds were characterized using microanalytical and spectroscopic (IR, MS, and NMR) data.
\end{abstract}

Keywords: Carbodiimides, nitrilium salts, 1,3,5-triazinium salt, cycloaddition

\section{Introduction}

The existence of nitrilium salts 1 (Figure 1) was predicted and nitrilium salts were proposed as intermediates in organic synthesis and reaction mechanisms by Hantzch $^{1}$ in 1931. Stable nitrilium salts, 1, were first prepared by Klages ${ }^{2}$ and Meerwein. ${ }^{3-5}$

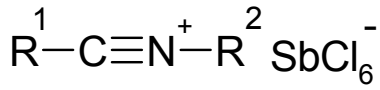

1<smiles>[R]N=C=N[Z]</smiles>

2

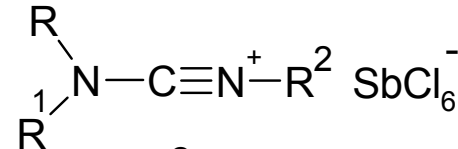

3

\section{Figure 1}

The dipolarophilicity of the nitrile moiety is only moderate. ${ }^{6}$ However, electron-withdrawing substituents or Lewis acid catalysis enhance the reactivity of nitriles towards nucleophiles and 1,3-dipolar species. ${ }^{6,7}$ Thus, nitrilium salts react smoothly with a variety of nucleophiles and 1,3dipoles. Cycloadditions of organic azides to nitrilium salts 1 lead to trisubstituted tetrazolium salts. ${ }^{8-11}$ These reactions are dominated by a concerted mechanism. ${ }^{9}$ However, the cycloaddition of the azide ion $\mathrm{N}_{3}{ }^{-}$to nitrilium ions is assumed to proceed via a two-step mechanism. ${ }^{9-13}$ Previously, we reported the synthesis of 1,2,4-oxadiazonium salts by the cycloaddition of nitrile oxides to nitrilium and cyanamidium salts. ${ }^{14}$ Also, the addition of nitrilium salts to $\alpha, \beta$ unsaturated carbonyl compounds led to the formation of $4 H-1,3-$ and $6 H-1,3$-oxazinium salts 
through a multistep reaction sequence starting with a [2+2] cycloaddition involving the carbonyl $\pi$-bond and the nitrilium functionality. ${ }^{15}$ Tetrahydrotriazinium and 2-azonia-allene salts were formed as a result of [2+2+2] cycloaddition of $\mathrm{N}$-alkylimines to nitrilium- and cyanamidium salts, ${ }^{16}$ while the azonia-allene salts were formed by 1-5 hydrogen shift. Abramovitch et al., ${ }^{17-20}$ and Jochims et al. ${ }^{21}$ reported the cycloaddition of heterocyclic nitrones to nitrilium salts, which was reviewed recently. ${ }^{22}$ 3,4-Bis-(methylthio)-2 $\mathrm{H}$-pyrrolium salts and 2-azonia-allenes were formed by the addition of nitrilium salts to bis-(methylthio)acetylene ${ }^{23}$ through the formation of 2-azonia-allene salts by a 1-5 hydrogen shift followed by cyclization. Moreover, 2,3,4,5tetrahydro-1,3,5-triazinium salts were obtained by the addition of $N$-substituted imines to nitrilium salts $1 .^{24}$ The formation of symmetrical and asymmetrical $N$-[4-imino-1,3-diazetidin-2ylidine]aminium hexachloroantimonate salts $\mathbf{4}$, as geometrical isomerization products upon the addition of carbodiimides $\mathbf{2}$ to cyanamidium salts $\mathbf{3}$ (shown in Scheme 1), have been reported quite recently. ${ }^{25}$

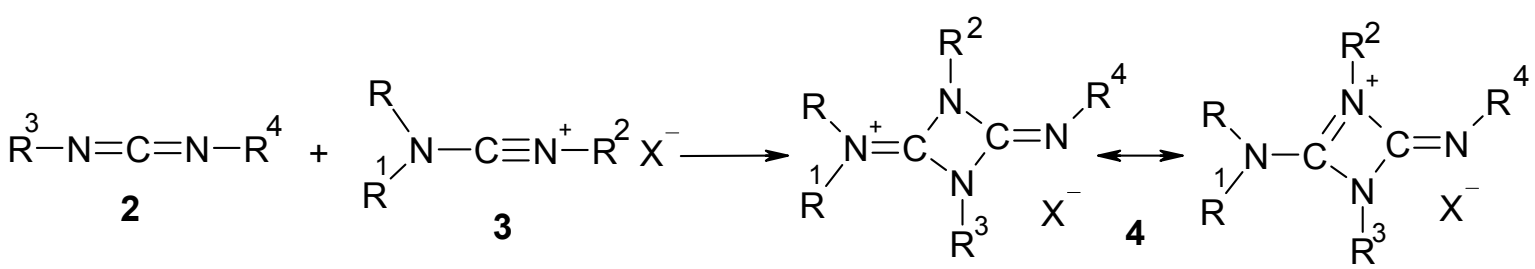

Scheme 1. Reaction of cyanamidium salts 3 and carbodiimides 2.

As part of ongoing studies related to of the synthesis and reactions of nitrilium-, $\mathbf{1}$, and cyanamidium, 2, salts, ${ }^{14-16,23-28}$ the reaction of carbodiimides $\mathbf{2}$ with $N$-alkylnitrilium salts $\mathbf{1}$ is reported herein, along with the resulting derivatives (Scheme 2), and their characterization.

\section{Results and Discussion}

The addition of the carbodiimides $\mathbf{2 a - d}$ to a solution of $N$-alkylnitrilium salts $\mathbf{1 a - f}$ in dichloromethane at low temperature afforded the triazinium salts $\mathbf{5 a - i}$ in $45-91 \%$ yield. It is suggested that the compounds $\mathbf{5 a}-\mathbf{i}$ are formed through a $[2+2+2]-$ cycloaddition, in analogy to the formation of cycloadducts by the reaction of nitrilium salts with $N$-substituted imines. ${ }^{25}$ The reaction involves the formation of intermediate 6 (1:1 adduct) which reacts further with another mole of the carbodiimide 2 (Scheme 3 ).

The structures of the newly prepared triazinium salts $\mathbf{5 a}-\mathbf{i}$ are derived from elemental analyses, and IR- and NMR- spectral data that are given in the Experimental Part. It is worth noting that the ${ }^{1} \mathrm{H}$ - NMR spectrum of 5e in $\mathrm{CD}_{3} \mathrm{CN}$ shows four signals for different isopropyl groups, and only one $N$-methyl group at $\delta=3.01$. Considerable line broadening of the ${ }^{13} \mathrm{C}$-signal of the $N$-methyl resonance indicates slow geometrical isomerization of the exocyclic $\mathrm{C}^{2}=\mathrm{N}$ and 
$\mathrm{C}^{4}=\mathrm{N}$ double bonds. Also, the ${ }^{13} \mathrm{C}$-NMR spectrum shows signals at $\delta=137.4,140.7$ and 165.5 ppm which are attributed to $\mathrm{C}^{2}=\mathrm{N}, \mathrm{C}^{4}=\mathrm{N}$ and $\mathrm{C}^{6}=\mathrm{N}^{+}$, respectively. The IR spectrum of $\mathbf{5 e}$ in $\mathrm{CH}_{2} \mathrm{Cl}_{2}$ shows stretching absorption peaks at $1690,1650,1540$ and $1500 \mathrm{~cm}^{-1}$ corresponding to different types of imines and iminium cation. The formation of only one isomer for $\mathbf{5 i}$ can be explained by the preferred nucleophilicity of $N$-methyl- over the $N$-tert-butyl group as a result of the steric effect of the latter group. A similar assumption was reported for the addition of carbodiimides to cyanamidium salts, ${ }^{25}$ and in the dimerization of carbodiimides in the presence of alkylating reagents. ${ }^{29-33}$ Geometrical isomerization was also observed in the ${ }^{13} \mathrm{C}$ - NMR of some compounds such as $\mathbf{5 i}$ and $\mathbf{5 j}$.



Compounds 1a-h

\begin{tabular}{l|cccccc} 
Entry & $\mathbf{a}$ & $\mathbf{b}$ & $\mathbf{c}$ & $\mathbf{d}$ & $\mathbf{e}$ & $\mathbf{f}$ \\
\hline $\mathrm{R}^{1}$ & $\mathrm{Me}$ & $\mathrm{Et}$ & $i-\mathrm{Pr}$ & $\mathrm{Bz}$ & $\mathrm{Ph}$ & $\mathrm{Ph}$ \\
$\mathrm{R}^{2}$ & $i-\mathrm{Pr}$ & $i-\mathrm{Pr}$ & $i-\mathrm{Pr}$ & $i-\mathrm{Pr}$ & $i-\mathrm{Pr}$ & $\mathrm{Me}$
\end{tabular}

\section{Compounds 2a-d}

\begin{tabular}{l|lll} 
Entry & $\mathbf{a}$ & $\mathbf{b}$ & $\mathbf{c}$ \\
\hline $\mathrm{R}^{3}$ & $i-\mathrm{Pr}$ & $\square$ & $\mathrm{Me}$ \\
$\mathrm{R}^{4}$ & $i-\mathrm{Pr}$ & $\square$ & \\
& &
\end{tabular}

Compounds $\mathbf{5 a - i}$

\begin{tabular}{l|lllllllll} 
Entry & $\mathbf{a}$ & $\mathbf{b}$ & $\mathbf{c}$ & $\mathbf{d}$ & $\mathbf{e}$ & $\mathbf{f}$ & $\mathbf{g}$ & $\mathbf{h}$ & $\mathbf{i}$ \\
\hline $\mathrm{R}^{1}$ & $\mathrm{Me}$ & $\mathrm{Et}$ & $i-\operatorname{Pr}$ & $i-\operatorname{Pr}$ & $\mathrm{Ph}$ & $\mathrm{Bz}$ & $\mathrm{Ph}$ & $\mathrm{Ph}$ & $\mathrm{Ph}$ \\
$\mathrm{R}^{2}$ & $i-\operatorname{Pr}$ & $i-\operatorname{Pr}$ & $i-\operatorname{Pr}$ & $i-\operatorname{Pr}$ & $\mathrm{Me}$ & $i-\operatorname{Pr}$ & $i-\operatorname{Pr}$ & $i-\operatorname{Pr}$ & $i-\operatorname{Pr}$ \\
$\mathrm{R}^{3}$ & $i-\operatorname{Pr}$ & $i-\operatorname{Pr}$ & $i-\operatorname{Pr}$ & $\square$ & $i-\operatorname{Pr}$ & $i-\operatorname{Pr}$ & $i-\operatorname{Pr}$ & $\square$ & $\mathrm{Me}$ \\
$\mathrm{R}^{4}$ & $i-\operatorname{Pr}$ & $i-\operatorname{Pr}$ & $i-\operatorname{Pr}$ & $\square$ & $i-\operatorname{Pr}$ & $i-\operatorname{Pr}$ & $i-\operatorname{Pr}$ & $\square$ &
\end{tabular}

Scheme 2. Synthesis of 1,3,5-triazinium salts, 5 .

\section{Mechanism}

The nucleophilic addition to nitrilium ions has been reported by Hegarty and his co-workers. ${ }^{29}$ to be stereo-electronically controlled. The nitrogen lone-pair always develops anti- to the intruding nucleophile ( $Z$-isomer). In the resulting imines, the nucleophile and the $\mathrm{N}$-substituent are synoriented with respect to each other. It is likely, therefore, that the addition of the carbodiimide 2 
to a nitrilium ion 1 gives the $(Z)$ adduct as the primary product. Thermal isomerization around the $\mathrm{R}-\mathrm{C}=\mathrm{N}-\mathrm{R}$ bond furnishes the $(E)$ form, which may readily undergo ring closure.

The formation of $\mathbf{5}$ by the addition of the carbodiimide $\mathbf{2}$ to the nitrilium salt $\mathbf{1}$ is believed to be due to stepwise polar cycloadditions. ${ }^{29,30}$ This involves the addition of two moles of carbodiimides 2 (added in two successive additions) via the formation of new cyanimidium salts 6 and 7 (Scheme 3). The latter intermediate, 7, isomerizes to the $(E)$ - geometrical isomer $\mathbf{8}$, followed by ring closure to form the $[2+2+2]$ cycloadduct, salt $\mathbf{5}$. This pathway is in contrast to the formation of a [2+2] cycloadduct, as in the case of dimerization of carbodiimides, ${ }^{29-31}$ and the case of the addition of carbodiimides to cyanamidium salts. ${ }^{25}$ The driving force for the second addition of another molecule of carbodiimide $\mathbf{2}$ to form 5 (instead of ring closure to diazetidinium salts) is presumably due to the formation of a resonance stabilized $[2+2+2]$ adduct $\left(5 \leftrightarrow 5^{\prime}\right)$.

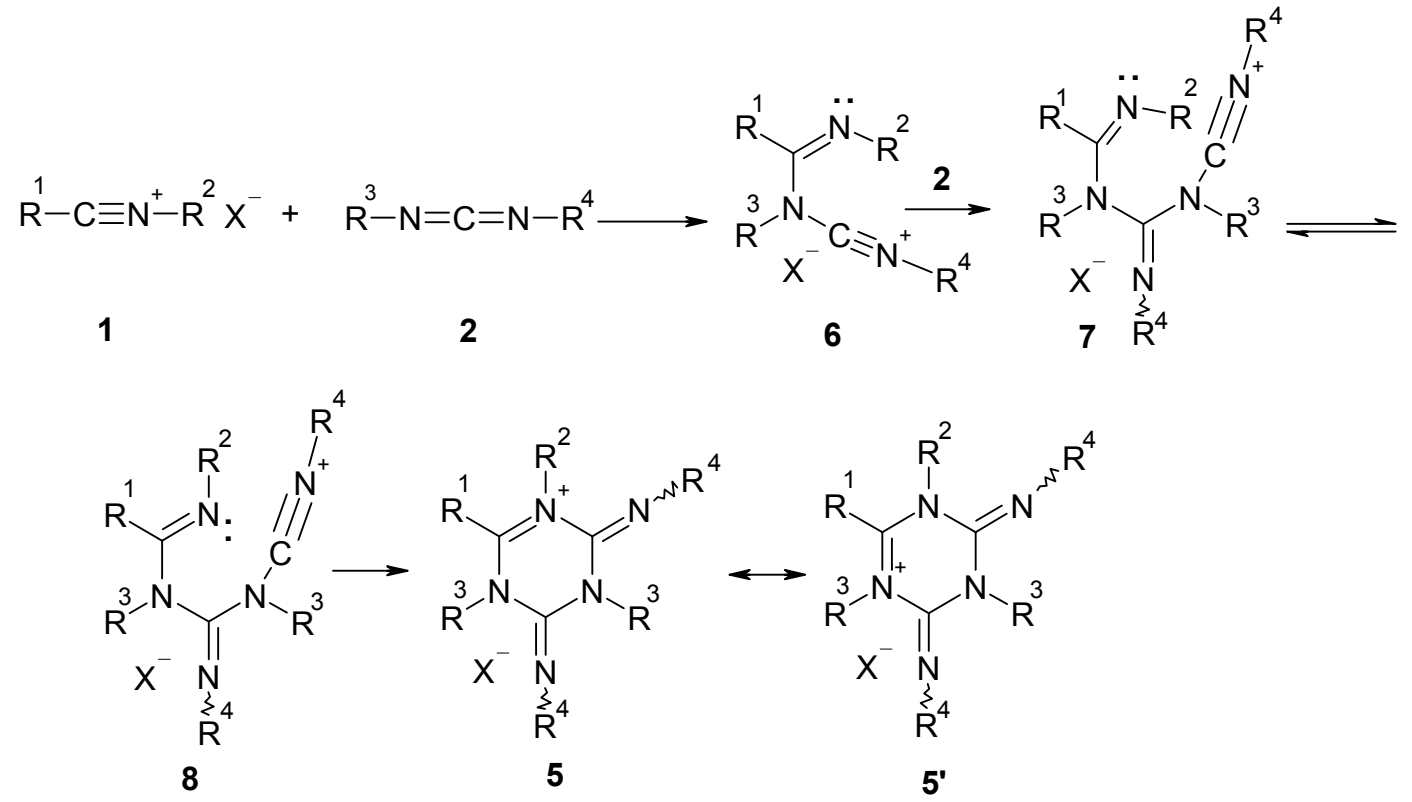

Scheme 3. Proposed mechanism for the formation of compounds 5.

\section{Experimental Section}

General Procedures. All experiments were carried out with the exclusion of moisture, in solvents dried by standard methods. Melting points were determined with Electrothermal 9100 apparatus and have not been corrected. The ${ }^{1} \mathrm{H}$ - and ${ }^{13} \mathrm{C}-\mathrm{NMR}$ spectra were recorded on Bruker AC-250 and Bruker DPX-300 instruments, using TMS as internal standard, and with deuterated chloroform, dichloromethane or acetonitrile as the solvent; chemical shifts $(\delta)$ are in ppm and coupling constants $(J)$ in Hz. IR spectra were recorded on a Perkin-Elmer 1600 FT-IR in $\mathrm{CH}_{2} \mathrm{Cl}_{2}$ solution; the frequencies are expressed in $\mathrm{cm}^{-1}$. Elemental microanalyses were obtained on an 
Elemental Analyzer (Carlo Erba 1106) from vacuum-dried samples. The nitrilium salts 1a, ${ }^{28}$ $\mathbf{1 b},{ }^{23} \mathbf{1 c},{ }^{28} \mathbf{1 d},{ }^{34} \mathbf{1 e},{ }^{4}$ and $\mathbf{1 f},{ }^{2}$ were prepared according to literature procedures.

General procedure for preparation of substituted 2,3,4,5-tetrahydro-2,4-diimino-1,3,5triazin-1-ium hexachloroantimonate, 5a-i

A solution of $10 \mathrm{mmol}$ of the carbodiimide 1a-c dissolved in $20 \mathrm{~mL}$ of dichloromethane was added dropwise to a cooled $\left(-20{ }^{0} \mathrm{C}\right)$, stirred solution of $5 \mathrm{mmol}$ of the appropriate nitrilium hexachloroantimonate $\mathbf{2 a - f}$. The solution was stirred at this temperature for $1 \mathrm{~h}$, then allowed to rise to $10{ }^{\circ} \mathrm{C}$ and stirred for an additional $1 \mathrm{~h}$, after which time the IR of the solution showed the disappearance of the absorption of the nitrilium peak of the nitrilium salts $\left(2230 \mathrm{~cm}^{-1}\right)$. The reaction solution was reduced to half volume in vacuo, then cooled to $-20{ }^{0} \mathrm{C}$, then $100 \mathrm{~mL}$ of absolute diethyl ether was added dropwise to give a powder precipitate, which was collected under vacuum and dried. The precipitate was purified by recrystallization from $10 \mathrm{~mL} / 100 \mathrm{~mL}$ diethyl ether.

1,3,5-Tri-isopropyl-2,4-di-isopropylimino-6-methyl-2,3,4,5-tetrahydro-1,3,5-triazin-1-ium hexachloroantimonate (5a). White solid (70\%); mp 108-110 ${ }^{\circ} \mathrm{C}$; IR $\left(\mathrm{CH}_{2} \mathrm{Cl}_{2}\right) v_{\max }\left(\mathrm{cm}^{-1}\right)$ : 3051, 2968, 1774, 1716; ${ }^{1} \mathrm{H}$ NMR (300 MHz, $\left.\mathrm{CD}_{3} \mathrm{CN}\right): \delta 1.19$ (d, $\left.J=6.9 \mathrm{~Hz}, 12 \mathrm{H}, 2 \mathrm{x}\left(\mathrm{CH}_{3}\right)_{2} \mathrm{CH}\right), 1.25$ (d, J=6.9 Hz, 6H, $\left.\left(\mathrm{CH}_{3}\right)_{2} \mathrm{CH}\right), 1.57\left(\mathrm{~d}, J 6.9 \mathrm{~Hz}, 12 \mathrm{H}, 2 \mathrm{x}\left(\mathrm{CH}_{3}\right)_{2} \mathrm{CH}\right), 2.69\left(\mathrm{~s}, 3 \mathrm{H}, \mathrm{CH}_{3}\right), 3.25$ (sept., J 6.9 Hz, 2H, $\left(\mathrm{CH}_{3}\right)_{2} \mathrm{CH}$ ), 4.28 (sept., J 6.9 Hz, 1H, $\left(\mathrm{CH}_{3}\right)_{2} \mathrm{CH}$ ), 4.568 (sept., J 6.9 Hz, 2H, $\left.2\left(\mathrm{CH}_{3}\right)_{2} \mathrm{CH}\right) ;{ }^{13} \mathrm{C}$ NMR $\left(75 \mathrm{MHz}, \mathrm{CD}_{3} \mathrm{CN}\right): \delta$ (ppm) 20.8, 21.4, 22.7, 24.9, 50.6, 55.5, 56.6, 136.2, 136.3,167.6. Anal. Calcd. for $\mathrm{C}_{19} \mathrm{H}_{38} \mathrm{~N}_{5} \mathrm{SbCl}_{6}$ (671.0): C, 34.01; H, 5.71; N, 10.44. Found: C, 34.19; H, 5.68; N, 10.23\%.

6-Ethyl-1,3,5-tri-isopropyl-2,4-di-isopropylimino-2,3,4,5-tetrahydro-1,3,5-triazin-1-ium hexachloroantimonate (5b). White solid $(66 \%)$; mp $125-128^{\circ} \mathrm{C} ; \mathrm{IR}\left(\mathrm{CH}_{2} \mathrm{Cl}_{2}\right) v_{\max }\left(\mathrm{cm}^{-1}\right): 1690$ (w), 1655 (m), 1520 (s). ${ }^{1} \mathrm{H}$ NMR (300 MHz, CD $\left.{ }_{3} \mathrm{CN}\right): \delta(\mathrm{ppm}) 1.13$ (d, J $6.9 \mathrm{~Hz}, 12 \mathrm{H}$, 2( $\left.\left.\mathrm{CH}_{3}\right)_{2} \mathrm{CH}\right), 1.22\left(\mathrm{~d}, J 6.9 \mathrm{~Hz}, 6 \mathrm{H},\left(\mathrm{CH}_{3}\right)_{2} \mathrm{CH}\right), 1.35\left(\mathrm{~d}, J 7.3 \mathrm{~Hz}, 3 \mathrm{H}, \mathrm{CH}_{3} \mathrm{CH}_{2}\right), 1.56(\mathrm{~d}, J 6.9$ $\mathrm{Hz}, 12 \mathrm{H}, 2\left(\mathrm{CH}_{3}\right)_{2} \mathrm{CH}$ ), 2.94 (q, J $7.3 \mathrm{~Hz}, 2 \mathrm{H}, \mathrm{CH}_{3} \mathrm{CH}_{2}$ ), 3.15 (sept., $J 6.9 \mathrm{~Hz}, 2 \mathrm{H}, 2\left(\mathrm{CH}_{3}\right)_{2} \mathrm{CH}$ ), 4.36 (sept., J 6.9 Hz, 1H, $\left.\left(\mathrm{CH}_{3}\right)_{2} \mathrm{CH}\right), 4.58$ (sept., $\left.J 6.9 \mathrm{~Hz}, 2 \mathrm{H}, 2\left(\mathrm{CH}_{3}\right)_{2} \mathrm{CH}\right) .{ }^{13} \mathrm{C} \mathrm{NMR}(75 \mathrm{MHz}$, $\left.\mathrm{CD}_{3} \mathrm{CN}\right): \delta(\mathrm{ppm})$ 11.8, 20.3 (br), 21.2, 23.5, 24.7, 50.6, 55.6, 56.7, 136.3, 136.4,167.5. Anal. Calcd. for $\mathrm{C}_{20} \mathrm{H}_{40} \mathrm{~N}_{5} \mathrm{SbCl}_{6}$ (685.1): C, 35.07; H, 5.89; N, 10.22. Found: C, 35.22; H, 5.67; N, $10.38 \%$.

1,3,5,6-Tetra-isopropyl-2,4-di-isopropylimino-2,3,4,5-tetrahydro-1,3,5-triazin-1-ium hexachloroantimonate (5c). White solid (53\%); mp 137-139 ${ }^{\circ} \mathrm{C}$; IR $\left(\mathrm{CH}_{2} \mathrm{Cl}_{2}\right) v_{\max }\left(\mathrm{cm}^{-1}\right)$ : 1700, 1650, 1600 (w), 1525 (s), 1400 (br), 1370, 1200, 1100. ${ }^{1} \mathrm{H}$ NMR $\left(\mathrm{CDCl}_{3}\right): \delta 1.21$ (d, 12H, J 7.0 Hz, $\left.2\left(\mathrm{CH}_{3}\right)_{2} \mathrm{CH}\right), 1.65\left(3 \mathrm{~d}, 18 \mathrm{H}, J 7.0 \mathrm{~Hz}, 3\left(\mathrm{CH}_{3}\right)_{2} \mathrm{CH}\right), 1.8\left(\mathrm{~d}, 6 \mathrm{H}, J 7.0 \mathrm{~Hz},\left(\mathrm{CH}_{3}\right)_{2} \mathrm{CH}\right), 3.18$ (sept., $2 \mathrm{H}, J 7.0 \mathrm{~Hz}, 2\left(\mathrm{CH}_{3}\right)_{2} \mathrm{CH}$ ), 3.87 (sept., $\left.1 \mathrm{H}, J 7.0 \mathrm{~Hz},\left(\mathrm{CH}_{3}\right)_{2} \mathrm{CH}\right), 4.30$ (sept., 2H, J 7.0 Hz, $\left.2\left(\mathrm{CH}_{3}\right)_{2} \mathrm{CH}\right), 4.97$ (sept., $\left.1 \mathrm{H}, J 7.0 \mathrm{~Hz},\left(\mathrm{CH}_{3}\right)_{2} \mathrm{CH}\right) .{ }^{13} \mathrm{C} \mathrm{NMR}\left(\mathrm{CDCl}_{3}\right): \delta 19.5,20.9,21.9,23.3$, 30.7, 50.6, 55.23, 56.3, 137.5, 137.6, 172.5. Anal. Calcd. for $\mathrm{C}_{21} \mathrm{H}_{42} \mathrm{~N}_{5} \mathrm{SbCl}_{6}$ (699.1): C, 36.05; H, 6.06; N, 10.02. Found: C, 35.99; H, 5.92; N, 9.91\%. 
3,5-Dicyclohexyl-2,4-dicyclohexylimino-1,6-di-isopropyl-2,3,4,5-tetrahydro-1,3,5-triazin-1ium hexachloroantimonate (5d). White solid (45\%); mp 139-141 ${ }^{\circ} \mathrm{C}$; IR $\left(\mathrm{CH}_{2} \mathrm{Cl}_{2}\right) v_{\max }\left(\mathrm{cm}^{-1}\right)$ : 1690, 1660, 1600 (w), 1520 (s), 1400 (br), 1200. ${ }^{1} \mathrm{H}$ NMR $\left(\mathrm{CDCl}_{3}\right): \delta$ 1.21-1.82 (m, 20H), 1.67 $\left(\mathrm{d}, 6 \mathrm{H}, J 6.9 \mathrm{~Hz},\left(\mathrm{CH}_{3}\right)_{2} \mathrm{CH}\right), 1.75\left(\mathrm{~d}, 6 \mathrm{H}, J 6.9 \mathrm{~Hz},\left(\mathrm{CH}_{3}\right)_{2} \mathrm{CH}\right), 2.42(\mathrm{~m}, 2 \mathrm{H}), 2.66(\mathrm{~m}, 1 \mathrm{H}), 3.93$ $(\mathrm{m}, 2 \mathrm{H}), 4.40(\mathrm{~m}, 1 \mathrm{H}) .{ }^{13} \mathrm{C} \mathrm{NMR}\left(\mathrm{CDCl}_{3}\right): \delta 128.6,137.2,157.1$, and others; the spectrum changed during measurements. Anal. Calcd. for $\mathrm{C}_{33} \mathrm{H}_{58} \mathrm{~N}_{5} \mathrm{SbCl}_{6}(859.3)$ : C, 46.12; H, 6.80; N, 8.15. Found: C, 46.22; H, 7.05; N, 7.92\%.

3,5-Di-isopropyl-2,4-di-isopropylimino-1-methyl-6-phenyl-2,3,4,5-tetrahydro-1,3,5-triazin1-ium hexachloroantimonate (5e). White solid (63\%); mp 158-161 ${ }^{\circ} \mathrm{C}$; IR $\left(\mathrm{CH}_{2} \mathrm{Cl}_{2}\right) v_{\max }\left(\mathrm{cm}^{-}\right.$ $\left.{ }^{1}\right): 1690(\mathrm{~m}), 1650$ (s), 1540 (s), 1500 (w), 1400 (br). ${ }^{1} \mathrm{H}$ NMR $\left(\mathrm{CD}_{3} \mathrm{CN}\right): \delta 1.14$ (d, 6H, J 7.0 Hz, $\left.\left(\mathrm{CH}_{3}\right)_{2} \mathrm{CH}\right), 1.24\left(\mathrm{~d}, 6 \mathrm{H}, J 7.0 \mathrm{~Hz},\left(\mathrm{CH}_{3}\right)_{2} \mathrm{CH}\right), 1.48\left(\mathrm{~d}, 6 \mathrm{H}, J 7.0 \mathrm{~Hz},(\mathrm{CH})_{2} \mathrm{CH}\right), 1.97(\mathrm{~d}, 6 \mathrm{H}, J$ $7.0 \mathrm{~Hz},\left(\mathrm{CH}_{3}\right)_{2} \mathrm{CH}$ ), 3.01 (s, 3H, $\mathrm{CH}_{3}$ ), 3.54 (sept., 1H, J $6.9 \mathrm{~Hz},\left(\mathrm{CH}_{3}\right)_{2} \mathrm{CH}$ ), 3.94 (sept., $1 \mathrm{H} J 6.9$ $\mathrm{Hz},\left(\mathrm{CH}_{3}\right)_{2} \mathrm{CH}$ ), 4.30 (sept., $1 \mathrm{H}, J=7.0 \mathrm{~Hz},\left(\mathrm{CH}_{3}\right)_{2} \mathrm{CH}$ ), 4.40 (sept., $\left.1 \mathrm{H}, J=6.9 \mathrm{~Hz},\left(\mathrm{CH}_{3}\right)_{2} \mathrm{CH}\right)$, 7.54-7.78 (m, 5H). ${ }^{13} \mathrm{C}$ NMR $\left(\mathrm{CD}_{3} \mathrm{CN}\right): \delta$ 19.4, 21.4, 22.6, 23.31, 23.6, 24.1, $38.8\left(\mathrm{CH}_{3}-\mathrm{N}^{+}\right)$, 50.4, 51.1, 57.7, 59.2, 118.4, 126.6, 126.7, 127.8, 131.1, 133.4, 137.4, 140.7, 165.5. Anal. Calcd. for $\mathrm{C}_{22} \mathrm{H}_{36} \mathrm{~N}_{5} \mathrm{SbCl}_{6}(705.1)$ : C, 37.48; H, 5.15; N, 9.93. Found: C, 37.59; H, 5.34; N, 9.75\%.

6-Benzyl-1,3,5-tri-isopropyl-2,4-di-isopropylimino-2,3,4,5-tetrahydro-1,3,5-triazin-1-ium hexachloroantimonate (5f). Yellow solid $(67 \%)$; mp $115-120{ }^{\circ} \mathrm{C}$; IR $\left(\mathrm{CH}_{2} \mathrm{Cl}_{2}\right) v_{\max }\left(\mathrm{cm}^{-1}\right)$ : $1690(\mathrm{w}), 1650,1590,1520(\mathrm{~s}) .{ }^{1} \mathrm{H} \mathrm{NMR}\left(\mathrm{CD}_{3} \mathrm{CN}\right): \delta 1.18\left(\mathrm{~d}, 12 \mathrm{H}, J=6.9 \mathrm{~Hz}, 2\left(\mathrm{CH}_{3}\right)_{2} \mathrm{CH}\right)$, $1.21\left(\mathrm{~d}, 6 \mathrm{H}, J=6.9 \mathrm{~Hz},\left(\mathrm{CH}_{3}\right)_{2} \mathrm{CH}\right), 1.49$ (d, 6H, J 6.9 Hz, 2(CH3) $\left.{ }_{2} \mathrm{CH}\right), 3.31$ (sept., 2H, J 6.9, $\left.2\left(\mathrm{CH}_{3}\right)_{2} \mathrm{CH}\right), 4.36$ (sept., $\left.2 \mathrm{H}, J 6.9 \mathrm{~Hz}, 2\left(\mathrm{CH}_{3}\right)_{2} \mathrm{CH}\right), 4.42\left(\mathrm{~s}, 2 \mathrm{H}, \mathrm{CH}_{2}\right), 4.47$ (m, 2H, $\left.2\left(\mathrm{CH}_{3}\right)_{2} \mathrm{CH}\right), 7.26(\mathrm{~m}, 2 \mathrm{H}), 7.50(\mathrm{~m}, 3 \mathrm{H}) ;{ }^{13} \mathrm{C} \mathrm{NMR}\left(\mathrm{CD}_{3} \mathrm{CN}\right): \delta 21.1(\mathrm{br}), 21.4,23.5,36.5,50.7$, 55.5, 57.7, 128.3, 129.2, 130.6, 131.9, 132.0, 138.0, 166.2. Anal. Calcd. for $\mathrm{C}_{25} \mathrm{H}_{42} \mathrm{~N}_{5} \mathrm{SbCl}_{6}$ (747.12): C, 40.19; H, 5.67; N, 9.38. Found: C, 40.24; H, 5.44; N, 9.21\%.

\section{1,3,5-Tri-isopropyl-2,4-di-isopropylimino-6-phenyl-2,3,4,5-tetrahydro-1,3,5-triazin-1-ium}

hexachloroantimonate (5g). White solid (91\%); mp 166-168 ${ }^{\circ} \mathrm{C}$; IR $\left(\mathrm{CH}_{2} \mathrm{Cl}_{2}\right) v_{\max }\left(\mathrm{cm}^{-1}\right): 1690$ (w), 1660, 1510 (s), 1400. ${ }^{1} \mathrm{H}$ NMR $\left(\mathrm{CD}_{3} \mathrm{CN}\right): \delta 1.21\left(\mathrm{~d}, 12 \mathrm{H}, J 5.7 \mathrm{~Hz}, 2\left(\mathrm{CH}_{3}\right)_{2} \mathrm{CH}\right) ; 1.27$ (d, $\left.6 \mathrm{H}, J 6.4 \mathrm{~Hz},\left(\mathrm{CH}_{3}\right)_{2} \mathrm{CH}\right) ; 1.41\left(\mathrm{~d}, 6 \mathrm{H}, J 6.0 \mathrm{~Hz}\right.$ ); 3.47 (sept., $\left.2 \mathrm{H}, J 6.4 \mathrm{~Hz}, 2\left(\mathrm{CH}_{3}\right)_{2} \mathrm{CH}\right) ; 3.86$ (sept., 1H, J $\left.6.7 \mathrm{~Hz},\left(\mathrm{CH}_{3}\right)_{2} \mathrm{CH}\right) ; 4.40$ (sept., $\left.2 \mathrm{H}, J 6.2 \mathrm{~Hz}, 2\left(\mathrm{CH}_{3}\right)_{2} \mathrm{CH}\right) ; 7.71$ (m, 3H); 7.74 (m, $2 \mathrm{H}) ;{ }^{13} \mathrm{C}$ NMR $\left(\mathrm{CD}_{3} \mathrm{CN}\right): \delta 21.4,21.5,23.5,51.0,56.9,59.6,126.4,128.3,131.5,133.4,133.5$, 137.9, 165.8. Anal. Calcd. for $\mathrm{C}_{24} \mathrm{H}_{56} \mathrm{~N}_{5} \mathrm{SbCl}_{6}$ (733.1): C, 39.32; H, 5.50; N, 9.55. Found: C, $39.42 ; \mathrm{H}, 5.67 ; \mathrm{N}, 9.56 \%$.

3,5-Dicyclohexyl-2,4-dicyclohexylimino-1-isopropyl-6-phenyl-2,3,4,5-tetrahydro-1,3,5triazin-1-ium hexachloroantimonate (5h). White solid $(63 \%)$; mp 159-161 ${ }^{\circ} \mathrm{C}$; IR $\left(\mathrm{CH}_{2} \mathrm{Cl}_{2}\right)$ $v_{\max }\left(\mathrm{cm}^{-1}\right): 1690(\mathrm{w}), 1650(\mathrm{~m}), 1600(\mathrm{w}), 1400(\mathrm{br}) ;{ }^{13} \mathrm{C} \mathrm{NMR}\left(\mathrm{CDCl}_{3}\right): \delta 23.8,23.9,24.7$, 25.0, 25.1, 25.6, 26.1, 31.3, 33.2, 58.3, 58.4, 59.1, 63.4, 67.43, 125.3, 126.5, 131.1, 133.0, 136.0, 136.3, 165.0. Anal. Calcd. for $\mathrm{C}_{36} \mathrm{H}_{56} \mathrm{~N}_{5} \mathrm{SbCl}_{6}$ (893.3): C, 48.40; H, 6.32; N, 7.70. Found: C, 48.19; H, 6.49; N, 7.70\%.

2,3-Di-tert-butylimino-1-isopropyl-3,5-dimethyl-6-phenyl-2,3,4,5-tetrahydro-1,3,5-triazin-1ium hexachloroantimonate (5i). White solid (64\%); mp 137-139 ${ }^{\circ} \mathrm{C}$; IR $\left(\mathrm{CH}_{2} \mathrm{Cl}_{2}\right) v_{\max }\left(\mathrm{cm}^{-1}\right)$ : 
1700, 1670, 1600, 1550, 1400 (br). ${ }^{1} \mathrm{H}$ NMR $\left(\mathrm{CD}_{2} \mathrm{Cl}_{2}\right): \delta 1.44\left(\mathrm{~s}, 9 \mathrm{H},\left(\mathrm{CH}_{3}\right){ }_{3} \mathrm{C}\right), 1.47(\mathrm{~d}, 6 \mathrm{H}, J=$ $\left.6.8 \mathrm{~Hz},\left(\mathrm{CH}_{3}\right)_{2} \mathrm{CH}\right), 1.49\left(\mathrm{~s}, 9 \mathrm{H},\left(\mathrm{CH}_{3}\right)_{3} \mathrm{C}\right), 3.08\left(\mathrm{~s}, 3 \mathrm{H}, \mathrm{CH}_{3}\right), 3.23\left(\mathrm{~s}, 3 \mathrm{H}, \mathrm{CH}_{3}\right), 3.97$ (sept., 1H, $\left.J 6.8 \mathrm{~Hz},\left(\mathrm{CH}_{3}\right)_{2} \mathrm{CH}\right), 7.49-7.78(\mathrm{~m}, 5 \mathrm{H}) .{ }^{13} \mathrm{C} \mathrm{NMR}\left(\mathrm{CD}_{2} \mathrm{Cl}_{2}\right): \delta$ 21.8, 29.2, 29.8, 30.6, 31.2, 39.4, 45.7, 56.0, 57.6, 58.9, 126.5, 127.4, 127.9, 129.2, 131.0, 133.1, 133.3, 133.4, 134.5, 165.4; Anal. Calcd. for $\mathrm{C}_{21} \mathrm{H}_{42} \mathrm{~N}_{5} \mathrm{SbCl}_{6}$ (699.1): C, 36.08; H, 6.06; N, 10.02. Found: C, 35.99; H, 5.92; N, $9.91 \%$.

\section{Acknowledgements}

This work is supported by Al al-Bayt University. Financial support from DAAD and DFG (Germany) is gratefully acknowledged. The author is indebted to Prof. Jäger (Stuttgart University), Prof. Laatsch (Goettingen University), Prof J. C. Jochims (Konstanz University) and Prof. M Al-Abadela and Dr. J. Zahra (Jordan University) for their kind encouragement, advice and criticism.

\section{References}

1. Hantzsch, A. Ber. 1931, 64, 667.

2. Klages, F.; Grill, W. Liebigs Ann. Chem. 1955, 594, 21.

3. Meerwein, H. Angew. Chem. 1955, 67, 374.

4. Meerwein, H.; Laasch, H.; Mersch, R.; Spille, J. Chem. Ber. 1956, 89, 209.

5. Meerwein, H.; Laasch, H.; Mersch, R.; Nentwig, J. Chem. Ber. 1956, 89, 224.

6. Bast, K.; Christl, M.; Huisgen, R.; Mack, M. Chem. Ber. 1972, 105, 2825.

7. Chang, M. S.; Lowe, J. U. J. Org. Chem. 1967, 32, 1577 (1967).

8. Morrocchi, S.; Ricca, A.; Velo, L. Tetrahedron Lett. 1967, 8, 331.

9. Quast, H.; Bieber, L. Tetrahedron Lett. 1976, 17, 1485.

10. Quast, H.; Bieber, L.; Meichsner, G. Chem. Ber. 1987, 120, 469.

11. Artamonova, T. V.; Zhivich, A. V.; Dubinskii, M. Yu.; Koldobskii, G. I. Synthesis 1996, 1428.

12. Carboni, B.; Carrie, R. Tetrahedron 1984, 40, 4115.

13. Kevill, D. N.; Weitl, F. L. J. Org. Chem. 1970, 35, 2526.

14. Abu-El-Halawa, R.; Shrestha-Ddwadi, P. B.; Jochims, J. C. Chem. Ber. 1993, 126, 107.

15. Abu-El-Halawa, R.; Glocker, M. O.; Zsolnai, L.; Huttner, G.; Jochims, J. C. Synthesis 1990, 763.

16. Abu-El-Halawa, R. Al-Manarah 1999, 4, 189.

17. Abramovitch, R. A.; Singer, G. M. J. Am. Chem. Soc. 1969, 91, 5672.

18. Abramovitch, R. A; Rogers, R. B. Tetrahedron Lett. 1971, 12, 1951.

19. Abramovitch, R. A.; Singer, G. M. J. Org. Chem. 1975, 39, 1795. 
20. Abramovitch, A.; Shinkai, I. Acc. Chem. Res. 1976, 9, 192.

21. Hitzler, M. G.; Freyhardt C. C.; Jochims, J. C. J. Prakt. Chem. Chemikar-Zeitung 1996, 338, 243.

22. Youssif, S. ARKIVOC 2001, (i), 242.

23. Abu-El-Halawa, R.; Jochims, J. C. Synthesis 1992, 871.

24. Abu-El-Halawa, R. Al-Manarah 1998, 3, 53.

25. Abu-El-Halawa, R. J. Saudi Chem. Soc. 2008, 12, in press.

26. Abu-El-Halawa, R.; Wirschum, W.; Mustafa, H.; Jochims, J. C. J. Prakt. Chem. 1996, 338, 598.

27. Mustafa, A. H.; Abu-El-Halawa, R.; Wirschum, W. Jochims, J. C. J. Prakt. Chem. 1997, 339,615 .

28. Jochims, J. C.; Abu-El-Halawa R. Chem. Ber. 1984, 117, 1900.

29. Hegarty, A. F. Acc. Chem. Res. 1980, 13, 448, and references cited therein.

30. Schmidt R. R. Angew. Chem. 1973, 85, 235, Angew. Chem. Int. Ed. 1973, 12, 212.

31. Hartke, K.; Rossbach, F.; Radau, M. Liebigs Ann. Chem. 1972, 762, 167.

32. Hartke, K.; Rossbach, F.; Radau, M. Angew. Chem. 1968, 80, 83; Angew. Chem. Int. Ed. 1968, 7, 72 .

33. Scheffold, R.; Saladin, E. Angew. Chem. 1972, 84, 158; Angew. Chem. Int. Ed. 1972, 11, 229.

34. Jochims, J. C.; Abu-El-Halawa, R. Synthesis 1990, 488. 